\title{
Better appreciating the scale of it (Lemaitre and de Sitter at the BAAS Centenary)
}

\author{
Siska De Baerdemaeker*and Mike D. Schneider ${ }^{\dagger}$
}

\begin{abstract}
In September 1931, a panel discussion was convened at Central Hall Westminster on the subject of the 'Evolution of the Universe', at the centenary meeting of the British Association for the Advancement of Science. Center stage was what to do about the evolving universe being younger than the stars, evidently a paradox in the relativistic study of the evolving universe, at the time. Here, we discuss two diametrically opposed reactions to the paradox, which were each broadcast at the meeting by Lemaitre and de Sitter, respectively. As we argue, that both could be projected to the public as viable reflects an unsettled question at the foundations of the then-nascent discipline: what is the role for considerations of scale in relativistic cosmology?
\end{abstract}

\section{Acknowledgements}

We foremost would like to thank Richard Dawid, George Gale, Don Howard, Casey McCoy, John Norton, and Chris Smeenk, as well as our two anonymous referees, for their helpful draft comments. We are also grateful for the audiences at Stockholm University and at the Center for Philosophy of Science at University of Pittsburgh. We are indebted to the Archives Georges Lemaitre, both for their friendliness and for making it so easy to access their records online, especially during the height of the COVID-19 pandemic in Europe. The seeds for this project were planted during the 2018 Rotman Summer Institute in Philosophy of Cosmology in Goderich (informally known as summer camp for philosophy of cosmology); we are grateful to the organizers for helping build a community in history and philosophy of cosmology where collaborations like ours can flourish. Partial funding for this research was provided by the Swedish Research Council, project number 1598801 (Siska) and by the Templeton Foundation grant "New Directions in Philosophy of Cosmology", grant number 61048 (Mike).

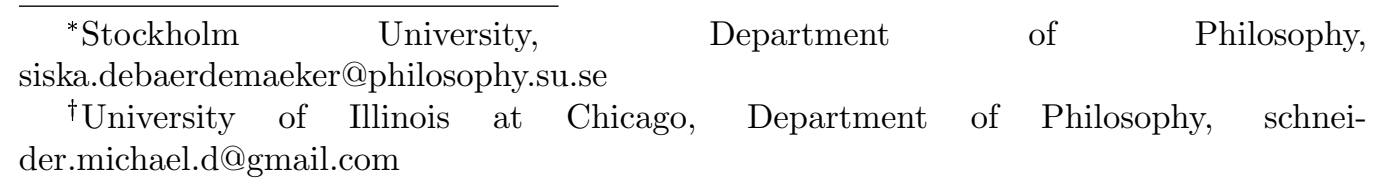




\section{Introduction}

In September 1931, the British Association for the Advancement of Science (BAAS) convened their centenary meeting in London. As remarked by Jan Christiaan Smuts, then-incoming president of the BAAS, in his inaugural address at the meeting: "[The BAAS] had for its two main objects the fostering of intercourse among workers in science, and the creation of a platform for propaganda purposes from which the progress and discoveries in science could be brought to notice of the public. [...] The Association has served its purpose admirably as an effective sounding board of the scientific advance." (British Association for the Advancement of Science, 1932, xix-xx) In other words, one of the primary aims of the BAAS - one which, by the organization's own estimation, had been consistently achieved thus far- was to provide an interested public with ready access to the frontiers of scientific research.

It is juxtaposed against this mission of the BAAS that we turn to the disjointed panel discussion on the 'Evolution of the Universe' that was conducted at that centenary meeting. A central point of debate was the recent materialization of a paradox: the evolving universe itself was estimated to be too young to accommodate the age of the oldest stars. This 'timescale problem' presented a looming challenge for the then-nascent study of the evolving universe. The expert perspectives shared in the BAAS discussion showcase some of the radically different responses: some faced the paradox head-on, as in need of resolution, while others embraced it as a fact of the matter. In the following, our goal is to show that both approaches were viable at the time because there was an unsettled question at the foundation of the discipline concerning the role that considerations of scale stand to play in cosmological theorizing. While it may have been agreed that the physics of systems existing at smaller scales should matter in cosmological research, there were multiple perspectives present in the community about how the knowledge of such physics should be made to matter in furthering that research. Indeed, it was not even agreed which physical quantity's magnitude ought to define the scale of their subject matter, in relation to that of other physics- e.g. length/time or weight.

In Section 2, we will argue that the context of the BAAS meeting motivates a particular reading of the rampant intellectual disunity exhibited 
across the prepared remarks of the expert participants in the discussion. ${ }^{1}$ Namely, despite various points of contact between each of their perspectives, there was a mere minimal consensus view that could be ascribed to the community, as a whole. The relativistic study of the evolving universe was, at the time, immature. But then, in light of this historical interpretation, we may proceed to read the record of the discussion as evidence of some of what was up for grabs in the foundations of the community's theorizing. To this effect, we focus in the latter half of the paper on the remarks prepared by Georges Lemaitre and Willem de Sitter. Close readings of each of their arguments - Sections 3 and 4, respectively ${ }^{2}$ - draw out the different explanatory roles played by the physics of other scales in each of their responses to the timescale problem.

\section{2 'Chaos among the Scientists'}

The goal of this section is to place the 1931 discussion at the BAAS in its proper context, in order to motivate the readings of Lemaitre's and de Sitter's remarks that are given in sections 3 and 4. Toward this end, we will briefly rehearse some of the intellectual pre-history of the then-nascent relativistic study of the evolving universe, ${ }^{3}$ as well as provide details about the event itself (including its reception by the public).

\subsection{The evolving universe}

Between 1923 and 1929, two major developments in astronomy came out of the Mount Wilson Observatory. In 1923, Edwin Hubble resolved the 'Great Debate' between Shapley and Curtis about the scale of the universe. By

\footnotetext{
${ }^{1}$ To get just some taste of what we mean by disunity, note that the discussion included topics as varied as heat death, empirical data on the characteristic profiles of cosmic rays, the theory of stellar evolution, and speculations about the physics of a primordial era of the expanding universe (not to mention the emergence of life and mind, and even a call for extraterrestrial alien diplomacy!).

${ }^{2}$ Our presentation reverses the order of their remarks in the report. This is a deliberate choice: for reasons that will become clear, we take de Sitter to take the more radical view on the relation between physics on different scales.

${ }^{3}$ Here, we draw primarily on the exposition of Kragh (2007)'s Chapter 3, but there is ample work on this time period, including (Nussbaumer and Bieri, 2009, 2011) as well as references cited throughout this paper.
} 
observing Cepheid variables in the Andromeda Nebula, Hubble established that it had to lie outside of the Milky Way. By 1929, Hubble had expanded his distance measurements to 24 galaxies. One upshot of the scale of the universe being, without much doubt, considerably larger than that of a galaxy is that the astronomical record now underdetermines whether the universe need be, on the whole, quasi-static (O'Raifeartaigh et al., 2014, fn. 16). Moreover, by 1929, Hubble had also obtained measurements for the radial velocities of 46 galaxies based on redshifts - many drawn from previous work by Vesto Slipher (O'Raifeartaigh, 2013), some made at Mount Wilson by Milton Humason. The results were consolidated into a paper entitled "A relation between distance and radial velocity among extra-galactic nebula", which Hubble published in March of that year. (By 1931, Hubble and Humason had expanded this data-set significantly.)

The dissemination of Hubble's distance-velocity relation caused a dramatic shift in relativistic cosmological theorizing. Following public remarks by de Sitter and Arthur Eddington about the need to explain this observed relationship, relativistic models were sought that could describe a spatially uniform universe that was not quasi-static (nor empty). Hence, previously unappreciated work by Alexander Friedman and by Georges Lemaître completed in the mid-1920s became important. Lemaitre was even asked to republish an English translation of his work (Livio, 2011), originally written in French, in the Monthly Notices of the Royal Astronomical Society (Lemaitre, 1931c).

In retrospect, the republication of Lemaittre's work in 1931, following Eddington's work in 1930 on the instability of Einstein's static universe (and in conjunction with Hubble and Humason's publication of the expanded dataset reinforcing the linearity of the distance-velocity relation), marks the emergence of a new discipline, dedicated to the relativistic study of the evolving universe. ${ }^{4}$ The evolving universe was that described by a closed and expanding model of general relativity, with a non-zero cosmological constant: the

\footnotetext{
${ }^{4}$ The perspective we take here about the importance of the years 1930-1931, leading up to the BAAS, in the early development of relativistic cosmology echos that suggested by Gale and Shanks (1996), which was, evidently, endorsed by W.H. McCrea in an interview late in life (cf. footnote 10 and surrounding discussion in the article). Similarly, O'Raifeartaigh (2013), in the first paragraph of the final section, describes the acceptance of the expansion of the universe as a "watershed in modern cosmology", and Kragh (1996, 32-33) calls the "fusion" of redshift observations with expanding universe models a "paradigm shift" in cosmology.
} 
Eddington-Lemaître model. According to the model, the universe was, for an indefinite amount of time in the past and to little consequence, static, whereupon at some point it abruptly began to expand, due to an instability precipitated by any notable change at galactic scales. Hubble's redshift data was taken as the main source of evidence for that expansion, including, e.g., that the abrupt change captured in the model had already occurred, such that a certain, definite amount of time had elapsed, ever since. ${ }^{5}$

The ability to pinpoint, based on the redshift data, just how much time had elapsed since the start of the expansion according to that model is what gave rise to the 'timescale problem'. This was, in many respects, the outstanding problem for a relativistic understanding of the evolving universe, at the time. The evolution measured within galaxies - concerning stellar processes - was long, whereas the evolution measured across galaxies was, comparatively, short. Based on the evidence, the evolving universe appeared to be younger than its constituent parts. ${ }^{6}$

Yet, as we will now argue based on the remarks given at the BAAS, the emerging discipline was much more heterogeneous in its foundations than is suggested by the characterization that has been given in this section. In particular, that the participants were all comfortable discussing their subject in terms of the timescale problem, given the Eddington-Lemaitre model, ought only to be understood as demonstrating a surface-level consensus about the subject of their study.

\subsection{The meeting}

The Centenary Meeting of the BAAS occurred in late September, 1931. The discussion on the evolving universe that was held there occurred on the penul-

\footnotetext{
${ }^{5}$ Kragh $(2007,164)$ describes alternative explanations for the observed redshifts, including tired light-hypotheses. None received broad support from the cosmology community. To be clear, here we are adopting a view of the discipline similar to that found in Gale and Shanks (1996), where cosmology as a sub-discipline comprised a small community at the time, which was well-represented at the BAAS. We recognize that the uptake of the expanding universe-hypothesis was more controversial in the broader physics community, including among astronomers (see for example the discussion of Hubble's own views in (Kragh and Smith, 2003)).

${ }^{6}$ It is worth flagging that systematic errors in the distance-measurements turned out to have fueled much of this history. Recognition of these systematic errors in the 1950s did away with the apparent contradiction between the data on redshifts and stellar evolution, respectively (Kragh, 2007, 190-195).
} 
timate day of the meeting, in the unusually prominent location of Central Hall Westminster. Ultimately, a record of the discussion was included as an appendix to the meeting's report, as well as in a supplemental issue of Nature (Nature, 1931).

Recall from the Introduction that the aims of the BAAS concerned, chiefly, the broadcasting of the frontiers of scientific research to an interested public. In this regard, the discussion on the evolving universe was a resounding success. ${ }^{7}$ As captured by the press in the months following the event, the topic of the discussion was seen as important for dissemination, the discussants convened were seen as the relevant experts, and the audience in attendance was large: "One of the outstanding events of the British Association meetings was a discussion on "The Evolution of the Universe," which was listened to for three hours with breathless interest by several thousand people in the Central Hall, Westminster. Sir Frank Dyson presided, and the platform of speakers included almost the whole galaxy of original thinkers on this immense subject. [...] They all contradicted one another and afforded an intense intellectual entertainment." 8,9

Note that the cosmological community itself was, at the time, as small as a couple dozen scholars (Gale and Shanks, 1996, fn. 5). In this respect, the audience turnout is particularly impressive: nearly everyone in attendance, it is safe to say, was present as a member of the public.

So, the discussion was a success, as measured in terms of audience and reception. This is important, because it permits us to scrutinize the reactions to the discussion as a means to excavate the public image of cosmology that was being presented by the experts. As is alluded to in the aforementioned

\footnotetext{
${ }^{7}$ This stands in stark contrast with the reception of Einstein's public lectures in Oxford earlier that year (Fox, 2018).

${ }^{8}$ Daily Times, January 9, 1932, "Unique Event in Scientific World. Centenary Meetings.", author not listed; retrieved from Archives de l'Université catholique de Louvain Archives Georges Lemaitre, BE A4006 FG LEM-1541

${ }^{9}$ Curiously, a similar metaphor shows up in another press's earlier coverage of the event (and note the gravitas accorded to the topic of the discussion): "Discussion on the Evolution of the Universe [...] Here is a subject which affects us all, perhaps even more gravely than the question of the gold standard [...] It is astonishing, but true, that there are some empty seats to be seen. Very astonishing indeed when we consider the galaxy of intellect on the platform. A very Milky Way of it." (The Daily Mail, September 30, 1931, "Is the Universe Merely an Ash-Heap?", Collinson Owen; retrieved from Archives de l'Université catholique de Louvain - Archives Georges Lemaitre, BE A4006 FG LEM1541)
} 
press reports, the content of the discussion was dappled. This is also the sentiment expressed forcefully in the introduction to the record of the discussion published by Nature: ${ }^{10}$ "The most profound significance of the discussion as a whole, however, lay in the fact that the various speakers not only had no common starting-point but also made no attempt to find one..." (H.D., 1931, 700) Nonetheless, one need not conclude from the discussion being dappled, as did the author of the introduction in Nature, that there is "the urgent need of defining what actually is the basis of modern physical theory" (700). Rather, it could be that such foundations are what, with time and effort, are understood to emerge out of practice, as proceeds in the absence of any definite principles.

One sees something like this alternative viewpoint in a review by a science writer, much later, of a French-language translation of the record:

The reader can see without difficulty: there were not two among them who discussed the same problem, who defended the same side of the general - and immense - question of the evolution of the Universe. And the reader won't draw a categorical conclusion about what some of the leading thinkers in physics on the problem of the Universe tell her within these powerfully interesting pages. Without a doubt, this document will not provide her a firm conclusion. But it will make for a succulent and fortifying dish, like one rarely encounters. Her science will, without a doubt, be enhanced, but she will perceive difficulties to overcome, contradictions to reconcile, and she will recognize without a doubt that in science, there is a lot unknown. But it is known what is not known: that which is essential. ${ }^{11}$

In other words, the goal of the BAAS may be seen as having been heartily achieved, precisely in virtue of the disunity exhibited amongst the expert participants in the discussion.

It is worth pausing to reflect on what this claim to success stands in opposition to. One can imagine a similar public event whose success is demonstrated by press reports and other reactions all expressing that the

\footnotetext{
${ }^{10}$ The authorship in the introduction is attributed to the initials "H. D.", which in secondary literature has been linked to Herbert Dingle.

${ }^{11}$ Feuilleton du Journal des Debats, January 26, 1933, "L'évolution de l'Univers", Henry de Varigny, original translation; retrieved from Archives de l'Université catholique de Louvain - Archives Georges Lemaître, BE A4006 FG LEM-1541.
} 
experts conveyed a consensus picture, both on the settled conceptual terrain and the areas for further research. Thus, precisely what was valuable about the discussion was that it revealed disagreement as something characteristic of the discipline, whereas the discipline might otherwise have appeared to champion consensus: "The upshot [...] seems to be that the scientists are in violent and apparently irreconcilable disagreement concerning a number of things that have been hitherto accepted with some complacency." ${ }^{12}$ All told, to a public audience numbering in the thousands, the vanguard of relativistic cosmologists successfully communicated just how much of the foundations of their research remained, in practice, unsettled. This is the view we will adopt here: inasmuch as the discussion at the centenary meeting of the BAAS was intended for the public, the disunity exhibited in the experts' remarks indicates that the relativistic study of the evolving universe was, at the time, immature. ${ }^{13}$ Consequently, the particulars of those remarks provide snapshots of what was, at the time, up for grabs concerning the foundations of the discipline.

It is important to flag that this view runs contrary to another reading of the discussion at the BAAS, which is advanced by Gale and Shanks (1996). They read the record of the discussion as evidence of consensus being reached during this same period ("That the session represented consensus is clearly revealed by the content of the presentations" (281)). By their reckoning, while there were differences in philosophical persuasion amongst the participants at the time, those differences only amounted to "undercurrents" of disputes, which were "lurking" (283). But, given the context discussed so far, it is difficult to understand in what sense the disputes may be claimed to have been "lurking"; they are, rather, front and center, clearly evident to the thousands in the audience. ${ }^{14}$

\footnotetext{
${ }^{12}$ Month, January 1932, "Chaos among the Scientists: Expounding Universal Theories", author not listed; retrieved from Archives de l'Université catholique de Louvain - Archives Georges Lemaître, BE A4006 FG LEM-1541.

${ }^{13}$ We discourage reading 'immature' here in a strong Kuhnian way - in terms of relativistic cosmology existing in a pre-paradigm period - because this risks redirecting focus toward a question of when relativistic cosmology exited that period. By contrast, our focus is on the content of the foundational debates that arose amidst the lack of consensus, for what those debates reveal about how the subject matter and discipline were understood, at the time.

${ }^{14}$ In personal correspondence, Gale has clarified that he agrees that "much -indeed most - of the elements of the beginnings of modern cosmology were in controversy at the time", but emphasized (based on oral reports) the significance of the new consensus about
} 
As we will discuss in the next two sections, a closer reading of at least two of the participants' remarks highlights a curious kind of disagreement, concerning how to effect further development of their field, in the face of the timescale problem. Namely, they disagree about how contemporary knowledge about various physical processes within our universe are to be brought to bear in relativistic cosmology. In the first case, Lemaitre argues from a thesis about theoretical unification across atomic weight scales to a solution to the timescale problem, in the course of ongoing research. In the second case, de Sitter argues from the fact of the timescale problem to a conclusion concerning the radical separation of spatiotemporal scales (again, in the course of ongoing research).

\section{Lemaître's view}

Lemaitre's core proposal at the BAAS ${ }^{15}$ was that the entire evolution of the universe could be modeled as a radioactive decay chain, starting from a single primeval atom and eventually leading to the radioactive elements still detectable on Earth (as well as the non-radioactive elements). This model reveals theoretical unification across atomic weight scales as an organizing principle in his rhetoric. By 'organizing principle', we mean something instrumental: a tool to guide theoretical inquiry aimed at resolving the timescale problem - a tool made more compelling because of independent arguments for the relevance of radioactivity to stellar and cosmological evolution.

Lemaittre begins his address at the BAAS by summarizing the EddingtonLemaitre model and explaining how this model leads to the timescale problem. Lemaitre explicitly identifies the beginning of the formation of stars

expanding universe models. It is a question of historical interpretation as to whether this new consensus extends beyond a mere common starting point for further studies of the universe, now in terms of the consequences of its evolving nature (as opposed to its previously assumed static nature). Based on the prepared remarks in the BAAS report and the press' assessment of the discussion, we submit that it does not.

${ }^{15} \mathrm{As}$ is mentioned below, Lemaître changed his model several times throughout his career - a first time already in 1932/1933. In 1931, Lemaitre presents parts of the model considered here on three different occasions: in a Letter to Nature from May, at the BAAS in September, and in a French paper in November. Our discussion is limited to the view Lemaitre advocates in 1931. The current section should therefore also not be read as providing a reconstruction of what motivates Lemaitre to develop this cosmological model - for that, we refer the reader to (Lambert, 2000; Kragh and Lambert, 2007; Kragh, 2012). 
(and structure more generally) with the beginning of the expansion of the universe in the Eddington-Lemaitre model: "I think that these results [on the cause of the beginning of the expansion] add much weight to the fact that the actual velocity of expansion fixes a limit to the time scale of the evolution, as we must rule out of our speculations every process which would start a premature expansion of space. [...] this brings almost complete chaos into the already chaotic problem of stellar evolution. A complete revision of our cosmological hypothesis is necessary, the primary condition being the test of rapidity." (British Association for the Advancement of Science, 1932, 606) The cause of the expansion in the background here is slow proto-stellar evolution, where stars are formed due to gravitational collapse of a so-called 'Laplace nebula' - a diffuse, uniform nebula filling up all of space. ${ }^{16}$ Any process of condensation in the Laplace nebula would disturb the equilibrium of the static universe and kick-start its expansion or contraction. This 'cosmogony' lay at the roots of the timescale problem; ${ }^{17}$ Lemaitre calls for a new one.

Lemaître proceeds to develop a "fireworks theory of evolution", where the current phase of slow expansion is merely the "ashes and smoke of bright but very rapid fireworks" (606). And "the key of the [timescale] problem" (606), according to Lemaitre, was the discovery of cosmic rays (despite their origins being a point of contention between Jeans and Millikan at the BAAS).

He first notes the high energy levels of cosmic rays. Only stars have comparable energy levels. But meanwhile, stellar atmospheres would prevent the escape of cosmic rays from them. Hence, cosmic rays must have escaped before the formation of the stellar atmosphere. The question, then, is how could cosmic rays escape during star formation?

It might appear as if Lemaître has dug himself into somewhat of a hole. There are now two challenges: resolving the timescale problem, and explaining how cosmic rays can escape during the early days of star formation. It

\footnotetext{
${ }^{16}$ Although Lemaître doesn't explicitly use the phrase 'Laplace nebula' at the BAAS, he does so elsewhere, e.g. in (Lemaître, 1931b), where he explicitly references Laplace and Kant.

${ }^{17}$ Similarly, Eddington concludes, in his own remarks at the BAAS discussion, that "the observed motions of the nebulae are genuine; so that we must accept this alarmingly rapid dispersal of the nebulae with its important consequences in limiting the time available for evolution." (British Association for the Advancement of Science, 1932, 588) In Eddington's view, like in Lemaître's, the timescale problem requires a new understanding of any evolutionary dynamics within the universe, including the galactic processes that were otherwise thought to be long-lived. As we discuss below, de Sitter disagrees.
} 
is at this point, approximately halfway through his address, that Lemaitre reveals his organizing principle with a rhetorical question: "Cosmogony is atomic physics on a large scale - large scale of space and time - why not large scale of atomic weight?" (607) That is, Lemaitre proposes that the physical processes governing the evolution of atoms (i.e., radioactive decay) also govern the evolution of stars and of the universe as a whole. ${ }^{18}$ (How this affects the relevance of spatiotemporal descriptions is unclear- see below.)

With theoretical unification across atomic weight scales on the table, Lemaitre first solves the cosmic ray puzzle. He points out that "cosmic rays are like the rays of radium" (607), and that it is therefore plausible that cosmic rays "escaped from a big scale super-radioactive disintegration, the disintegration of an atomic star, the disintegration of an atom of weight comparable to the weight of a star" (607). In order to test his theory on the origins of cosmic rays, Lemaitre calls for further study of the composition of cosmic rays: if they are composed of charged particles as well as photons, that would lend further support to their super-radioactive origins.

Next, Lemaittre embeds his cosmic ray account into the evolution of the universe as a whole (608). Here, it will be helpful to borrow from Lemaitre's popular science article published in November: "The world proceeded from the condensed to the diffuse... The universe-atom [or primeval atom] broke apart into fragments, each fragment into smaller pieces. Imagine for simplicity's sake that each fragmentation happened in equal pieces, there would have been two hundred sixty generations in order to arrive at the pulverization of matter in our poor small atoms, almost too small to break apart even further." (Lemaître, 1931b, 118, original translation) The (short-lived, cf. note 15) proposal is that of a radioactive decay chain, beginning with the disintegration of a so-called 'primeval atom' comprising all matter content in the universe. The decay products of the primeval atom themselves would form the so-called 'star-atoms'; ${ }^{19}$ their decay would be the start of a star's life and potentially even planet formation (Lemaitre mentions in

\footnotetext{
${ }^{18}$ Our focus here is on Lemaitre's remarks at the BAAS, but the central role of theoretical unification across atomic weight scales as an organizing principle receives further support from one of Lemaître's arguments for the universe being past-finite (Lemaître, 1931b, 116), i.e. the presence of radioactive elements on Earth suggesting a relatively young universe.

${ }^{19}$ Interestingly, the phrase 'star-atom' already appears at the very end of Eddington's Stars and Atoms $(1927,127)$. Eddington uses the term to indicate that at the end of a star's lifetime, "there is no real discontinuity between the organization of the atom and the organization of the star" (126).
} 
passing (119) that the earth potentially was ejected from the sun during its solar-atom phase), and cosmic rays and remaining radioactive elements are both signatures of different stages of this decay chain. After an initial rapid expansion phase, the universe would enter a quasi-stable static phase, with the stars distributed uniformly across. The formation of nebulae out of the distribution of stars would break the equilibrium, causing further expansion and further separation of stars and nebulae after their formation- along the lines of his expanding model from 1927 and empirically supported by redshift observations. ${ }^{20}$

Of course, Lemaitre recognizes that his model crucially depends on the existence of super-radioactive elements with atomic numbers much higher than those of uranium. In this way, his organizing principle of theoretical unification ultimately indicates a direction for future research. He refers to Jeans' work as giving "strong reasons for admitting the existence of atoms of considerably higher atomic weight than our actual dead atoms" (British Association for the Advancement of Science, 1932, 607). But despite his deference to Jeans, Lemaître admits that more theorizing is required. At the very end of his address, he calls for a development of "a theory of nuclear structure sufficient to be applied to atoms of extreme weights" (608).

Two final remarks are in order. First, as a resolution to the timescale problem, Lemaître recognizes that his radioactive decay-model could recover some stellar phenomenology (e.g. the mass-luminosity relation), but not all aspects of stellar evolution (e.g. energy equipartition, evolution with loss of mass along the Hertzsprung-Russell diagram) (608). By 1933, Lemaître had abandoned the decay of star-atoms and the subsequent formation of nebula out of stars ((Lemaître, 1934) models star formation once again as gravitational collapse of diffuse nebulae). This highlights that the extreme theoretical unification of the initial proposal was not a methodological requirement for cosmological modeling, according to Lemaître. Rather, theoretical unification across atomic weight scales suggested a simple scenario to account for a wide range of phenomena. When that scenario was deemed implausible (although Lemaître would adhere to the primeval atom for the rest of his life), theoretical unification lost its appeal as well.

Second, while Lemaitre's model unifies physics across different scales of

\footnotetext{
${ }^{20}$ More accurately, Lemaître assumes a coasting model- a then-new multistage generalization of the Eddington-Lemaitre model, empirically supported in the final stage of expansion by the redshift observations.
} 
atomic weight, marrying his proposal with relativistic - i.e. spatiotemporalmodels of the universe seems to be an afterthought. Across the three papers in 1931 that we have focused on, three different proposals emerge. In his Letter to Nature in May, Lemaitre stipulates that the notions of space and time are statistical notions that only become meaningful after the decay of the primeval atom: "Now, in atomic processes, the notions of space and time are no more than statistical notions. [...] If the world has begun with a single quantum, the notions of space and time would altogether fail to have any meaning at the beginning; they would only begin to have a sensible meaning when the original quantum had been divided into a sufficient number of quanta." (Lemaitre, 1931a, 706) At the BAAS in September, he insists that the primeval atom is spatially extended and sits at the start of time: "At the origin, all the mass of the universe would exist in the form of a unique atom; the radius of the universe, although not strictly zero, being relatively very small." (British Association for the Advancement of Science, 1932, 608) In his article in November, he identifies the primeval atom with the initial singular state: "We can conceive that space started with the primeval atom and that the beginning of space marked the beginning of time. The radius of space started from zero." (Lemaître, 1931b, 119, original translation) The fact that Lemaitre gives three different proposals across the three papers is indicative of how deep the challenge of introducing spatiotemporal scales runs. This is a challenge similar to that faced by Bohr in reconciling the quantum picture of the atom with a spatiotemporal one, and there is reason to think Lemaitre had this in mind (Kragh and Lambert, 2007, 460).

\section{4 de Sitter's view}

As a consequence of his focus on atomic weight, Lemaittre struggled with the relevance of space and time in his 'cosmogony'. de Sitter, unconcerned with the antecedent, focuses his entire remarks at the BAAS on the latter. He begins with a play on words: since he has been asked to be short in time, he concedes that he has, nonetheless, prepared remarks that are extraordinarily long in length.

The reason for this bit of humor, as quickly becomes clear, is pedagogical: to underscore the enormous scales operating in the background of cosmological theorizing, where the speed of light is taken to be $1 .^{21}$ And, from this

\footnotetext{
${ }^{21}$ Or, as he puts it, "the corresponding units of time and of space are so very widely
} 
lesson, de Sitter moves immediately into another. He provides an argument that the universe is extraordinarily long-lived. Namely, the time elapsed since the first galaxies first began their internal evolution and the emission of the signals presently observed in the most distant observable galaxies must be much larger than the time it took for light to have travelled from those galaxies to here. Otherwise, we would observe those most distant galaxies as being in an appreciably earlier state of their internal evolution.

But here, de Sitter pauses the lesson. Having introduced the notion that the universe is, from this perspective, very plausibly long-lived, he then stresses: "It suffices for my argument to define the 'beginning' as that state of the universe and its constituent parts which we are with our present knowledge and theories content to use as a starting point, beyond which we do not wish, or are not able, to extend our investigations." (British Association for the Advancement of Science, 1932, 584) In other words, he advocates for an operational definition of what is meant by the universe's beginning. Namely, the beginning is just that which is suitable for particular uses, in whatever are our contemporary theories of the universe. In the case of galactic processes, the beginning of the universe simply is that which is a long time gone, so that the observed galaxies that are farthest away are, in their seemingly distant past, nonetheless already very like our own galaxy is, today. But what about the beginning of the universe at the largest scales?

At this point, de Sitter shifts to the new subject at hand: the study of the evolving universe, which, he stresses, is better suited than any relativistic model of a quasi-static universe to represent the "actual universe" (584). He attributes this shift in relativistic cosmology to Lemaitre's theoretical work on the expansion of the universe, in which the observed radial motions of galaxies are rendered as the "pure effect of the inertia of these bodies" $(584) .{ }^{22}$

It is worth pausing on this characterization of Lemaitre's work, and specifically de Sitter's use of the word "pure". As de Sitter mentions shortly before in his remarks, it is important that, in order to represent the actual universe,

different in relation to actual phenomena" (British Association for the Advancement of Science, 1932, 583).

${ }^{22} \mathrm{As}$ is clarified shortly thereafter, he is particularly interested in the EddingtonLemaître model. But in (de Sitter, 1933a) he explicitly endorses the model that features an initial singular state. This change of view reflects a more sophisticated take on the scope of the cosmological theory, in relation to the rest of physics. 
a model of the evolving universe ought to be non-empty. ${ }^{23}$ In other words, the observations of galaxies amounted to evidence that the evolving universethe cosmologists' object of study - is not empty. And, as he stresses in an article a few years later, the virtue of Lemaitre's theoretical work is not merely to have provided an idiosyncratic relativistic dynamics, which dominates the gravitational effects of galaxies and accounts for those galaxies' radial motions - i.e. in terms of a new "force" that becomes relevant at cosmological scales (de Sitter, 1934a, 208). Rather, what is so appealing is that the galaxies' apparent radial motions, as assessed with respect to the choice of cosmic rest frame, are identified as mean-field effects of the same gravitational forces that are understood to mediate the dynamics relevant at smaller scales, between all of the same galaxies (now individuated as distinct, mutually gravitating bodies). It is this mean-field approximation that is likely being referenced, obliquely, in the term "pure." 24

Turning next to discuss the timescale problem, it is important to note that, contra Lemaitre and Eddington, de Sitter does not present the problem as one to be resolved, on threat of paradox:

Now if we adopt this theory of the expanding universe, it is very tempting to seek a connection between this expansion and the evolution of the material bodies constituting the universe, and to identify the beginning of the expansion with the beginning of that evolution. [...]

We thus, however reluctantly, come to the conclusion that the expansion of the universe on the one hand, and the evolution of stellar systems and stars on the other hand, are two different processes, taking place side by side, but without any apparent connection between them. The expansion has only been going on during an interval of time which is as nothing compared with the duration of the evolution. Leaving the oscillating universes, and

\footnotetext{
${ }^{23}$ For comparison, Hubble $(1929,173)$ refers to the distance-velocity relation as suggestive of a "de Sitter effect", familiar from the geodesic structure of the empty model introduced by de Sitter in 1917 .

${ }^{24}$ Though de Sitter does not invoke the term "mean-field approximation", the analogy is explicit in (de Sitter, 1934b, 600-601): "...thus "gravitation" may be defined as that property of matter by which it determines the metric of time-space. It only becomes of importance when we consider the universe on a small scale, concentrating our attention on the motions of individual particles instead of on the statistical average of a large number of particles."
} 
those that start from a zero radius, out of account, the universe may have been practically stationary at or very near its minimum size for an infinite time before starting to expand, or it may have contracted during an infinite time and after passing through a minimum a few thousand million years ago started to expand again. In both cases there appears to be no causal connection between the change of size of the universe as a whole and the evolution of the systems which it contains. (British Association for the Advancement of Science, 1932, 585-586)

de Sitter presents the timescale problem subjunctively, in terms of a conclusion it entails about the relationship between the contents of two contemporary physical theories: a theory of the universe at the largest scales, where the motions of galaxies are inertial against the mean field, and a theory of the universe at smaller scales, where gravitational interactions amongst galaxies are important. Namely, were the two processes causally connected (as in the canonical 'cosmogony' of the Eddington-Lemaitre model), the operational definition of the beginning of the universe, applied simultaneously to the theories of each, would entail a contradiction: the beginning of the universe would be non-unique in time. Ergo, the community ought to accept, against temptation, that there is no causal connection between the two different evolutionary processes, whose dynamics are associated, respectively, with the theory of galactic processes and the theory of what contains them.

But, per the discussion above about the role of the mean-field approximation in the theory, the "universe as a whole" is not merely a container for galaxies subject to their own dynamics. What de Sitter is advocating here is a thesis of the radical separation of scales: unlike in Lemaitre's thinking on the subject, changes in the universe at the scales of galaxies do not play the role of causes in descriptions at larger scales. The beginning of the universe, when considered at the scales of galaxies, is altogether a different thing than the beginning of the universe, when considered at cosmological scales. So, in the process of shifting our attention from the universe at small scales to large scales, what even is the age of the universe changes.

Anticipating the charge that embracing a causal disconnect between theories at different scales amounts to embracing paradox, de Sitter insists that "We must be prepared to allow this 'Universe' the freedom to have contradictory properties, like we have been forced to grant to the atom..." (586). So: paradox is to be avoided in reasoning about the actual universe - this is 
what grounds the move to the radical separation of scales, to avoid the contradiction inherent in there being a non-unique beginning. But, meanwhile, the 'Universe' (a term which is capitalized uniquely in this passage) just is something whose galaxies, evolving with respect to each other according to the dynamics of the evolving universe, are internally composed of something whose dynamics proceed on timescales much longer.

This is an audacious claim. Recall from the block quote a few pages earlier that in his endorsement of Lemaitre's expanding universe account of the redshift data, de Sitter is not wedded to the Eddington-Lemaitre 'cosmogony'. Rather, de Sitter's ambivalence about the status of the actual universe at cosmological scales prior to its current expansion- including whether it even exists - is secondary to his view about the de-coupling between evolution modeled on galactic and on cosmological scales. ${ }^{25}$ Regardless of one's preference for one expanding universe model or another, there is no causal connection between theories deployed at different scales.

If this reading is correct, we might expect to see de Sitter embrace such a separation of scales as a basis for further cosmological research. In fact, we see something just like this in a paper he wrote in 1933 about what there is to learn on the basis of the timescale problem. In this paper, we see a continuation of the view he advanced at the BAAS: "We must therefore accept the paradox that the stars are older than the universe, if by the "age of the universe" we mean the time elapsed since $y$ passed through its minimum. ${ }^{26}$ It has been shown, however, that this minimum must not be conceived as the "beginning of the world," but as a transitory episode in the history of the universe, so that there is nothing paradoxical left in the paradox." (de Sitter, 1933a, 632) In other words, there is nothing paradoxical, so long as we see fit to embrace an interpretation of the theory that renders the beginning of the universe, considered at cosmological scales, as a transitory episode, from the perspective of smaller scales.

What would give the community reason to embrace that interpretation? de Sitter clearly has in mind the breakdown of the mean-field approximation,

\footnotetext{
${ }^{25}$ We recognize that questions about multi-scale modeling have been extensively discussed in contemporary philosophy of science. Drawing parallels to that literature is beyond the scope of the current project.

${ }^{26}$ In context, $y$ is a normalized spatial volume. In his preferred models, its minimum is zero (and he states that he dislikes oscillating models). So, in retrospect, we may identify this interpretation of "age of the universe" as "the time elapsed since the initial singular state".
} 
as galaxies come into close approach (i.e. as one proceeds backward through cosmic time). He notes (631) that "This approximation is, of course, entirely sufficient so long as the mutual distances are large, but ceases to be an approximation when these distances become very small." He continues, further along the same page, that "The conception of a universe shrinking to a mathematical point at one particular moment of time $t=t_{0}$ must thus be replaced by that of a near approach of all galaxies during a short interval of time near $t=t_{0}$." Yet, concerning this near approach, "[t]he dimensions of the galaxies themselves are not directly effected" and "there is no reason to suppose that [this moment in cosmic history] is of any special importance in the evolution of the stars." So, the beginning of the universe, assessed at cosmological scales, refers to a transitory episode in the history of the universe - as assessed at smaller scales - whereby conditions subsequently came to support the relevant mean-field approximation, which would thereby render Hubble's redshift data as due to the inertial effects of the relevant galaxies.

Note that, on this view, the obvious demand on the theorist is to better understand the breakdown of the mean-field approximation, in particular near the initial singular state. Sure enough, the final section of the paper is dedicated to getting some traction on that problem (633-634), and is followed up in (de Sitter, 1933b). In effect, he is at pains to establish heuristic support for the conjectural claim that the initial singular state is an artifact of the symmetries that fall out of the mean-field approximation, when that approximation is taken to hold all the way back during the near approach of all galaxies.

As noted by Earman (1999), Eddington and Einstein also each considered, around the same time, the possibility that the singular state at $t=0$ was a consequence of idealization. ${ }^{27}$ But as described by Tolman $(1934,486)$ in his textbook treatment of the subject, de Sitter appears to be the only one to seriously attempt to show that the descriptive relevance of that singular state fades away, by the lights of the theory itself, as greater complexities in the gravitational field become perturbatively relevant, given the pairwise near-approaches of all galaxies, as $t \rightarrow 0$. This is, of course, a difficult mathematical question, and one that is generally understood to have remained open until the singularity theorems of the late 1960s.

\footnotetext{
${ }^{27}$ For Einstein's view, see (O'Raifeartaigh et al., 2015), p. 17 and fn. 33 especially, and (O'Raifeartaigh et al., 2018), section 5.1.
} 


\section{Concluding remarks}

The BAAS discussion convened and recorded in 1931 provides an invaluable window into relativistic cosmological practice at the time, in virtue of being intended for the public. That the participants in the discussion each, evidently, felt comfortable delivering their own perspectives on the structure and subject of their discipline suggests that they did not feel pressure to convey any consensus picture of their discipline. In this sense, the record of the discussion may be seen as evidence that the period of practice was immature.

Given this historical interpretation, we are inclined to go looking for evidence about what was 'fair game' at the time. It is in this respect that we noted the sharp difference in Lemaitre and de Sitter's respective proposals for what cosmologists are to do, in light of the paradox of seemingly contradicting timescales. What we have argued is that the joint viability of both of their responses - working to resolve the paradox versus biting the bullet - is due to an unsettled foundational question about the role of scale in cosmology. In particular, we see, on one hand, a plea to resolve the timescale problem by means of new physics, specifically by a proposal to develop nuclear physics for extreme atomic weights, thereby achieving a theoretical unification across atomic weight scales. On the other hand, we see an embrace of the radical separation of spatiotemporal scales, as a result of embracing the truth of the paradox, i.e. as a fact of the matter. In this case, an entirely different proposal for future research is recommended: the study of the formal breakdown of the mean-field approximation, as mutually gravitating astrophysical bodies come into pairwise near approach. (It is interesting to note that each proposal tracks a different reckoning with the initial singularity, also a subject under conceptual debate at the time.)

The different perspectives on scales from Lemaittre and de Sitter provide but one example of what was up for grabs, however. Participants at the BAAS disagreed both on 'scientific' and 'philosophical' points. Regarding the former, we note the debate between Jeans and Millikan on the nature of cosmic rays, or between Jeans and Milne on star formation. Regarding the latter, Jeans' application of the second law of thermodynamics to the evolution of the universe was vehemently criticized by Millikan. Meanwhile, Eddington offered his own argument as to how the local mass properties of the electron and the global property of the radius of the universe are coconstitutive. To reiterate: any apparent agreement among the discussants 
at the BAAS could, at best, be superficial. Our project is just a first step in mining that richness.

\section{References}

British Association for the Advancement of Science (1932). Report of the Centenary Meeting. London, 2330 September 1931. Office of the British Association. https://www.biodiversitylibrary.org/item/96080\#page/5/mode/1up.

de Sitter, W. (1917). On the relativity of inertia. remarks concerning Einstein's latest hypothesis. KNAW, Proceedings 19(II), 1217-1225.

de Sitter, W. (1933a). On the expanding universe and the time-scale. Monthly Notices of the Royal Astronomical Society 93(8), 628-34.

de Sitter, W. (1933b). On the motion and the mutual perturbations of material particles in an expanding universe. Bulletin of the Astronomical Institutes of the Netherlands 7, 97.

de Sitter, W. (1934a). On distance, magnitude, and related quantities in an expanding universe. Bulletin of the Astronomical Institutes of the Netherlands 7, 205.

de Sitter, W. (1934b). On the foundations of the theory of relativity, with special reference to the theory of the expanding universe. Proceedings of the Royal Academy (Amsterdam) 37, 597-601.

Earman, J. (1999). The Penrose-Hawking singularity theorems: History and implications. The expanding worlds of general relativity 7, 235-270.

Eddington, A. (1927). Stars and Atoms. Oxford: Oxford University Press.

Eddington, A. (1930). On the instability of Einstein's spherical world. Monthly Notices of the Royal Astronomical Society 49, 668-78.

Fox, R. (2018). Einstein in oxford. Notes and Records: the Royal Society Journal of the History of Science 72(3), 293-318. 
Gale, G. and N. Shanks (1996). Methodology and the birth of modern cosmological inquiry. Studies in History and Philosophy of Science-Part B 27(3), 279-296.

H.D. (1931). The evolution of the universe. Nature 128, 699-701.

Hubble, E. (1929). A relation between distance and radial velocity among extra-galactic nebulae. Proceedings of the National Academy of Sciences 15(3), 168-73.

Hubble, E. and M. L. Humason (1931). The velocity-distance relation among extra-galactic nebulae. The Astrophysical Journal 74, 43.

Kragh, H. (1996). Cosmology and Controversy: The Historical Development of Two Theories of the Universe. Princeton University Press.

Kragh, H. (2012). 'The wildest speculation of all': Lemaître and the primevalatom universe. In R. D. Holder and S. Mitton (Eds.), Georges Lemaître: Life, Science and Legacy, Volume 395 of Astrophysics and Space Science Library, pp. 23-39. Berlin, Heidelberg: Springer Berlin Heidelberg.

Kragh, H. and R. W. Smith (2003). Who discovered the expanding universe? History of science 41(2), 141-162.

Kragh, H. S. (2007). Conceptions of Cosmos. Oxford: Oxford University Press.

Kragh, H. S. and D. Lambert (2007). The context of discovery: Lemaître and the origin of the primeval-atom universe. Annals of Science 64(4), $445-470$.

Lambert, D. (2000). Un Atome d'Univers. La vie et l'œure de Georges Lemaître. Bruxelles: Lessius.

Lemaitre, G. (1931a). The beginning of the world from the point of view of quantum theory. Nature 127, 706.

Lemaitre, G. (1931b). The expansion of the universe (l'expansion de l'espace). Revue des Questions Scientifiques 4(20), 101-120. 
Lemaître, G. (1931c). A homogeneous universe of constant mass and increasing radius accounting for the radial velocity of extra-galactic nebulae. Monthly Notices of the Royal Astronomical Society 91, 483-490.

Lemaître, G. (1934). Evolution of the expanding universe. Proceedings of the National Academy of Sciences 20, 12-17.

Livio, M. (2011). The expanding universe: lost (in translation) and found. Nature 479, 171-173.

Nature (1931, October). Supplemental Issue: Contributions to a British Association Discussion on the Evolution of the Universe, Volume 128.

Nussbaumer, H. and L. Bieri (2009). Discovering the Expanding Universe. Cambridge University Press.

Nussbaumer, H. and L. Bieri (2011). Who discovered the expanding universe? arXiv preprint arXiv:110\%.2281.

O'Raifeartaigh, C. (2013). The contribution of v.m. slipher to the discovery of the expanding universe.

O'Raifeartaigh, C., B. McCann, W. Nahm, and S. Mitton (2014). Einstein's steady-state theory: an abandoned model of the cosmos. The European Physical Journal H 39(3), 353-367.

O'Raifeartaigh, C., M. O'Keeffe, W. Nahm, and S. Mitton (2015). Einstein's cosmology review of 1933: a new perspective on the einstein-de sitter model of the cosmos. The European Physical Journal H 40(3), 301-335.

O'Raifeartaigh, C., M. O'Keeffe, W. Nahm, and S. Mitton (2018). One hundred years of the cosmological constant: from "superfluous stunt" to dark energy. The European Physical Journal H 43(1), 73-117.

Tolman, R. C. (1934). Relativity, Thermodynamics and Cosmology. London: Clarendon Press (Oxford University Press). 